\title{
Quorum sensing as a potential target for increased production of rhamnolipid biosurfactant in Burkholderia thailandensis E264
}

\author{
Irorere U. Victor ${ }^{1} \cdot$ Michal Kwiencien $^{2} \cdot$ Lakshmi Tripathi $^{1} \cdot$ Diego Cobice ${ }^{1} \cdot$ Stephen McClean $^{1} \cdot$ Roger Marchant $^{1}$. \\ Ibrahim M. Banat ${ }^{1}$
}

Received: 9 April 2019 / Revised: 22 May 2019 / Accepted: 25 May 2019 /Published online: 21 June 2019

(C) The Author(s) 2019

\begin{abstract}
Burkholderia thailandensis E264 is a potential non-pathogenic substitute for producing rhamnolipid biosurfactant, replacing the pathogenic Pseudomonas aeruginosa. However, it has low rhamnolipid production and longer fermentation time. We have earlier suggested that media supplementation with exogenous quorum sensing (QS) molecules could lead to early onset of biosynthesis and increased rhamnolipid yield. Here, we assessed the effect of single, double or triple mutations in the various QS systems of $B$. thailandensis on rhamnolipid production, with the view to see which system(s) have the most impact on rhamnolipid yield and subsequently use the QS molecule to potentially increase yield in the wild-type $B$. thailandensis. The triple mutant strain had a rhamnolipid yield of $4.46 \pm 0.345 \mathrm{~g} / 1$ at $240 \mathrm{~h}$ of fermentation which was significantly higher than that of the wild type $(0.94 \pm 0.06 \mathrm{~g} / \mathrm{l})$, an unexpected outcome. To gain more insight as to how this might occur, we studied substrate metabolism and energy storage in the form of polyhydroxyalkanoate (PHA) by both the triple mutant and the wild type. We observed increased glycerol metabolism and reduced PHA production in the triple mutant compared with the wild type. Glycerol concentration at $240 \mathrm{~h}$ and maximum PHA productivity (g/gDCB) were $8.76 \mathrm{~g} / \mathrm{l}$ or $16.19 \mathrm{~g} / \mathrm{l}$ and $21.80 \%$ or $31.4 \%$ in either the triple mutant or the wild type respectively. Complementation of the triple-mutant cultures with exogenous QS molecules restored rhamnolipid production to similar levels as the wild type. QS therefore is a potential target for increased rhamnolipid production in B. thailandensis.
\end{abstract}

Keywords Rhamnolipid · Quorum sensing $\cdot$ Burkholderia thailandensis $\cdot$ Wild type $\cdot$ Triple mutant

\section{Introduction}

The term quorum sensing (QS) describes the cell-to-cell communication mechanism/s in bacteria, which produces

Electronic supplementary material The online version of this article (https://doi.org/10.1007/s00253-019-09942-5) contains supplementary material, which is available to authorized users.

Ibrahim M. Banat

im.banat@ulster.ac.uk

Irorere U. Victor

Irorere-V@ulster.ac.uk

Michal Kwiencien

kwiecienmichal@poczta.fm

Lakshmi Tripathi

L.tripathi@ulster.ac.uk

Diego Cobice

D.cobice@ulster.ac.uk coordinated behaviours within a bacterial population upon the establishment of a quorum. This is achieved through the production, sensing and response to chemical signalling molecules known as pheromones, quorumones or autoinducers
Stephen McClean

S.mcclean@ulster.ac.uk

Roger Marchant

R.marchant@ulster.ac.uk

1 School of Biomedical Sciences, Faculty of Life and Health Sciences, Ulster University, Coleraine BT52 1SA, Northern Ireland, UK

2 Centre of Polymer and Carbon Materials, Polish Academy of Sciences, 34, M. Curie-Skłodowska St, 41-819 Zabrze, Poland 
(AIs) (Dusane et al. 2010; Rutherford and Bassler 2012; Williams 2007). Bacteria that use this system produce and release these signal molecules into the environment, the accumulation of which increases with increasing bacterial population. Consequently, a threshold concentration is reached, leading to the interaction of the signal molecules with cognate receptors forming a signal transduction cascade that ultimately results in the expression or repression of QS regulated genes (Papenfort and Bassler 2016; Williams 2007).

QS-regulated processes are usually those that are costly or ineffective when carried out by a single bacterium, but become effective when carried out by a population of bacteria. This phenomenon has been described as one that enables a bacterial population to act as a multicellular organism and obtain benefits which are unachievable should they act alone (Bassler and Losick 2006). Well-known QS-regulated processes include bioluminescence, biofilm formation and secretion of virulence factors (Bassler and Losick 2006; Papenfort and Bassler 2016). Specifically, QS has been implicated in the regulation of various bacterial physiological processes including antibiotic resistance, antibiotic synthesis, production of exopolysaccharide and rhamnolipid synthesis (Bjarnsholt et al. 2005; Duerkop et al. 2009; Marketon et al. 2003; Nickzad and Déziel 2016; Nickzad et al. 2015).

In the last three decades, the effect of quorum sensing on rhamnolipid production has been extensively studied, particularly in strains of Pseudomonas aeruginosa (Ahmed et al. 2019). Three different autoinducers regulating rhamnolipid production have been identified in $P$. aeruginosa: Pseudomonas autoinducer 1 or PAI-1 (N-(3-oxododecanoylhomoserine lactones or 3-oxo-C12-HSLs), Pseudomonas autoinducer 2 or PAI-2 (N-butyryl-homoserine lactones or C4-HSLs) and Pseudomonas Quinolone Signal or PQS (2heptyl-3-hydroxy-4-quinolone) (Pearson et al. 1994; Pearson et al. 1995; Pesci et al. 1999). PAI-1 synthesis is under the control of the LasI-LasR QS system while the rhlI-rhlR QS system controls the synthesis of PAI-2; these 2 systems are known to directly control rhamnolipid biosynthesis in P. aeruginosa (Dusane et al. 2010). Although the PQS autoinducer does not belong to a specific QS system, it regulates rhamnolipid production by either direct or indirect regulation of the synthesis of $\mathrm{C}_{4}$-HSL or PAI-2 belonging to the rhlI-rhlR QS system (Dusane et al. 2010; McKnight et al. 2000).

Single or multiple mutations in genes responsible for the synthesis of the different autoinducers have been shown to result in the decrease or absence of rhamnolipid synthesis in P. aeruginosa (Pearson et al. 1997). Furthermore, the addition of either exogenous synthetic autoinducers or spent media containing high concentrations of autoinducers has been reported to result in increased rhamnolipid biosynthesis in P. aeruginosa (Nakata et al. 1998; Ochsner et al. 1994).
These results do show a direct correlation between rhamnolipid production and quorum sensing. It also suggests that quorum sensing is a major target for increasing rhamnolipid productivity in microbial strains, particularly in the early growth stages of Burkholderia thailandensis, as we have previously suggested (Irorere et al. 2018). However, very little is known about the effect of quorum sensing on rhamnolipid biosynthesis in Burkholderia species. Recent studies on $B$. glumae have shown that the mutant strain of $B$. glumae tof $l$, deficient in the synthesis of N-3-octanoylhomoserine lactone $\left(\mathrm{C}_{8}\right.$-HSL), has reduced rhamnolipid production. This shows that rhamnolipid production is positively regulated by QS in B. glumae (Nickzad et al. 2015).

However, to the best of our knowledge, there are no available reports on the effect of quorum sensing on rhamnolipid production in B. thailandensis. In 2009, Chandler et al. characterised the QS systems in B. thailandensis. They confirmed that 3 QS systems were present in $B$. thailandensis, similar to those in B. pseudomallei but different from B. glumae which has a single QS system. The 3 QS systems in B. thailandensis comprise 3 pairs of synthase/receptors and 2 orphan receptors with no known synthase (Chandler et al. 2009; Ulrich et al. 2004). The quorum sensing system one or QS-1 in B. thailandensis comprises btaI1 and btaR1 genes, coding for the synthesis of the synthase and transcriptional regulator BtaI1 and BtaR1 respectively, which respond to and produce the autoinducer N-octanoyl homoserine lactone $\left(\mathrm{C}_{8}\right.$-HSL), while the QS-2 system consists of the btaI2 and btaR2 genes coding for the Btal2 synthase and the BtaR2 transcriptional regulator that responds to and regulates the synthesis of both N-3-hydroxyldecanoyl homoserine lactones (3OHC10-HSL) and N-3-hydroxyloctanoyl homoserine lactone $\left(3 \mathrm{OHC}_{8}\right.$-HSL). Finally, the QS-3 system is made up of the btaI3 and btaR3 genes coding for Btal 3 and BtaR3 synthase and transcriptional regulator respectively, which respond to and regulate the biosynthesis of N-3-hydroxyloctanoyl homoserine lactone $\left(3 \mathrm{OHC}_{8}\right.$-HSL) (Chandler et al. 2009; Le Guillouzer et al. 2017; Tseng et al. 2016).

This present study is focused on the effect of quorum sensing mutations on rhamnolipid production in $B$. thailandensis E264. We report that unlike $P$. aeruginosa or B. glumae, quorum sensing-deficient mutants of $B$. thailandensis E264 have increased rhamnolipid production compared with the wild type.

\section{Materials and methods}

\section{Microbial strains}

Rhamnolipid production was carried out by Burkholderia thailandensis ATCC 700388. A list of all mutant strains used in this study is presented in Table S1. Mutant strains were 
kindly provided by Dr. Josephine Chandler from the University of Kansas and the University of Washington, USA. The method used for making quorum sensing mutant strains is a markerless allelic exchange method and is fully described by Chandler et al. (2009)). Upon receipt, strains were immediately sub-cultured in nutrient broth for $24 \mathrm{~h}$, after which cultures were transferred to cryovials containing $50 \%$ glycerol at a ratio of $1: 1$ and kept at $-80^{\circ} \mathrm{C}$ before use. Prior to use, fresh stocks were taken from the freezer and streaked on nutrient agar (Sigma-Aldrich, UK) plates, incubated for $24 \mathrm{~h}$ at $30^{\circ} \mathrm{C}$. Fresh nutrient agar plates were prepared every 2 weeks, and care was taken to avoid cross contamination.

\section{Rhamnolipid production}

Initial rhamnolipid production was carried out for all mutant strains and the wild type, including single (S $\Delta b t a I 1, \mathrm{~S} \Delta b t a I 2$ and $\mathrm{S} \Delta$ btaI3) double (D $\Delta$ btaI2 $\Delta$ btaI3) and triple (T $\Delta$ btaIl $\Delta$ btaI2 $\Delta$ btal3) mutants. Single colonies of respective strains were used to prepare a starter culture in nutrient broth (Sigma-Aldrich, UK), grown at $30{ }^{\circ} \mathrm{C}$ and $200 \mathrm{rpm}$, to an $\mathrm{OD}_{600}$ of approximately 2.3 . These were used to inoculate the rhamnolipid production media (nutrient broth, with $4 \%$ $(w / v)$ glycerol) in a 1-1 Erlenmeyer flask at a 10\% inoculum ratio. For rhamnolipid production, inoculated flasks were incubated at $30^{\circ} \mathrm{C}$ and $200 \mathrm{rpm}$ for $240 \mathrm{~h}$ with samples collected regularly to quantify rhamnolipid production, assess growth, measure surface tension and determine glycerol concentration. All experiments were carried out in biological triplicates, with additional flasks inoculated to quantify rhamnolipid concentration at different times of fermentation.

A complementation study was carried out on the triple

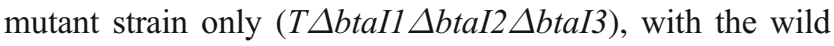
type used as control. Two different complementation analyses were carried out. The first involves growing the wild-type B. thailandensis E264 in nutrient broth for $24 \mathrm{~h}$ at $30{ }^{\circ} \mathrm{C}$ and $200 \mathrm{rpm}$. Cells were removed by aseptic centrifugation, and the supernatant was sterilised by filtration with a $0.25-\mu \mathrm{m}$ syringe filter (Sartorius Minisart Plus). No growth was observed on nutrient agar plates inoculated by spread plating $100 \mu \mathrm{l}$ of the filtered spent medium. Fifty millilitres of the filtered spent medium was added to a 40-ml sterile fresh $\mathrm{nu}-$ trient broth containing $4 \mathrm{~g}$ glycerol; this was inoculated with a $10-\mathrm{ml}$ starter culture, giving a $4 \%(w / v)$ carbon source concentration.

The second complementation involved supplementation of the fermentation nutrient broth (containing $4 \%$ glycerol) with $4 \mu \mathrm{M}$ each of synthetic $3 \mathrm{OHC}_{10}$-HSLs, $3 \mathrm{OHC}_{8}$-HSLs and $\mathrm{C}_{8^{-}}$HSLs (Cambridge Bioscience, UK). The medium was inoculated with $10 \%(v / v)$ inoculum concentration. All complementation studies were carried out in triplicate with the wild-type strain used as a control.

\section{Rhamnolipid extraction and purification}

To quantify rhamnolipid produced during fermentation, $30 \mathrm{ml}$ of fermentation broth was taken and centrifuged for $15 \mathrm{~min}$ at $13,000 \mathrm{~g}$. Biomasses were separated from the supernatant by transferring the supernatant to new $50-\mathrm{ml}$ tubes, and rhamnolipid extraction was carried out by acid precipitation and solvent extraction as previously described (Irorere et al. 2018; Smyth et al. 2014). This involves reducing the $\mathrm{pH}$ of the supernatant to 2.0 followed by three subsequent extractions with ethyl acetate. Anhydrous $\mathrm{MgSO}_{4}$ was used to remove the residual aqueous phase from the organic phase containing rhamnolipid. This was followed by filtration, rotary evaporation of solvent, transfer of rhamnolipid to vials and removal of any residual solvent with nitrogen gas. The weight of the extracted rhamnolipid was determined gravimetrically.

Extracted rhamnolipid were purified prior to characterisation by solid phase extraction (SPE), using Strata SI-1 Silica (55- $\mu \mathrm{m}, 70-\mathrm{A})$ Giga tubes (Phenomenex) as previously described (Irorere et al. 2018).

\section{HPLC-MS analysis of rhamnolipid}

After purification, the determination of the relative proportions of rhamnolipid congeners present in each sample was carried out by HPLC-MS. The system used consisted of a classic LCQ ion trap mass spectrometer (Thermo Finnigan, UK) coupled to a SpectraSYSTEM LC P4000 (Thermo Finnigan, UK) highperformance liquid chromatography system, equipped with a

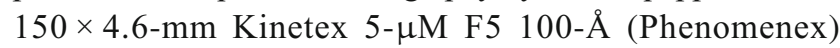
reversed-phase LC column. The mass spectrometer was operated at a flow rate of $0.8 \mathrm{ml} / \mathrm{min}$ in the negative ionisation mode with ion spray voltage of $4500 \mathrm{~V}$, capillary voltage of $-25 \mathrm{~V}$ and capillary temperature of $250{ }^{\circ} \mathrm{C}$. The chromatography was conducted in gradient mode, using water and acetonitrile as mobile phases A and B respectively and a sample injection volume of $10 \mu$. The gradient profile used is shown in Table S2.

For quantification, purified rhamnolipid was used as a standard to create a calibration curve (Fig. S1). Quantification was performed in single ion recording (SIR) mode using normalised internal standard (stearic acid). Different pseudomolecular ions representing monorhamnolipid and dirhamnolipid were initially used as quantifier ions, to study their linearity with increasing rhamnolipid concentrations. The pseudomolecular ions $733 \mathrm{~m} / \mathrm{z}, 705 \mathrm{~m} / \mathrm{z}$ and $615 \mathrm{~m} / \mathrm{z}$ gave the best linearity and were subsequently used for quantification analysis.

\section{Determination of microbial growth and surface tension}

Microbial growth was determined by measuring the optical density of the culture medium $\left(\mathrm{OD}_{600}\right)$ at regular intervals 
during the fermentation using a Novaspec II spectrophotometer (Pharmacia Biotech). Media with $\mathrm{OD}_{600}$ values higher than 1 were diluted, and the results obtained were multiplied by the dilution factor. Values obtained were used to plot growth curves to determine the differences in growth patterns of the strains used. For comparison, viable counts of the triple mutant and wild-type strains were performed at various times during the fermentation using the Miles and Misra technique (Miles et al. 1938).

Surface tension measurements were also carried out at intervals during the fermentation as a measure of biosurfactant production. Measurements were carried out on a KRUSS KIOST tensiometer with a platinum ring using 10-ml supernatants from the fermentation broth.

\section{Measurement of glycerol concentration}

Glycerol concentration was measured during fermentation using a spectrophotometric method for the determination of free glycerol concentration (Bondioli and Della Bella 2005). This method involves the formation of formaldehyde from glycerol through a series of reaction involving periodate oxidation. The formaldehyde produced is then converted to 3,5-diacetyl-1,4dihydrolutidine via Hantzsch's reaction in the presence of acetylacetone and ammonium acetate (Bondioli and Della Bella 2005).

Cell-free supernatant samples were diluted in a working solvent which consisted of 1:1 $\mathrm{v} / \mathrm{v}$ distilled water and $95 \%$ ethanol. Two millilitres of diluted sample was then transferred to a $10-\mathrm{ml}$ tube followed by the addition of $1.2 \mathrm{ml}$ of $10 \mathrm{mM}$ sodium periodate solution. The solution was manually mixed by shaking for $30 \mathrm{~s}$ before adding $1.2 \mathrm{ml}$ of a $0.2-\mathrm{M}$ acetylacetone solution. This was then transferred to a water bath maintained at $70{ }^{\circ} \mathrm{C}$ for $1 \mathrm{~min}$, stirring manually. Samples were immediately cooled by immersing tubes in a beaker of tap water after the reaction time. The optical density of the samples was determined at $410 \mathrm{~nm}$ using a Novaspec II spectrophotometer (Pharmacia Biotech), using a blank prepared by using $2 \mathrm{ml}$ of working solvent. A standard curve was also prepared using different concentrations of glycerol (BDH Chemicals).

Both sodium periodate and acetylacetone solutions were prepared daily and used immediately after preparation. Sodium periodate solution was prepared by dissolving $21 \mathrm{mg}$ of sodium meta-periodate (Sigma, UK) in $5 \mathrm{ml}$ of 1.6 $\mathrm{M}$ acetic acid, followed by the addition of $5 \mathrm{ml}$ of $4.0 \mathrm{M}$ ammonium acetate (Sigma, UK), while acetylacetone solution was prepared by first mixing $5 \mathrm{ml}$ of $1.6 \mathrm{M}$ acetic acid with $5 \mathrm{ml}$ of $4.0 \mathrm{M}$ ammonium acetate, followed by the addition of $200 \mu \mathrm{l}$ acetylacetone.

\section{Polyhydroxyalkanoate extraction}

Polyhydroxyalkanoate (PHA) extraction was carried out as described previously (Tripathi et al. 2012) with slight modification, from biomass obtained by centrifugation of a $30-\mathrm{ml}$ fermentation broth at $13,000 \mathrm{~g}$ for $15 \mathrm{~min}$. Biomasses were frozen at $-20{ }^{\circ} \mathrm{C}$ and then lyophilised for $48 \mathrm{~h}$. The cell dry weight of the biomass was recorded; PHA was extracted from lyophilised cells by adding $10 \mathrm{ml}$ of chloroform to cells at $95^{\circ} \mathrm{C}$ for $4 \mathrm{~h}$. Cells were filtered off, and the PHA was extracted using a 10-fold volume of ice-cold methanol. The precipitated PHAs were collected by centrifugation and dried under a stream of nitrogen gas to constant weight.

\section{PHA characterisation}

Nuclear magnetic resonance Extracted polymers were identified by nuclear magnetic resonance (NMR) analysis. The NMR spectra were recorded using a Bruker Avance II $600 \mathrm{MHz}$ with Ultrashield Plus Magnets. The ${ }^{1} \mathrm{H}$ and ${ }^{13} \mathrm{C}$ NMR spectra were run in $\mathrm{CDCl}_{3}$ using tetramethylsilane (TMS) as an internal standard.

Differential scanning calorimetry Differential scanning calorimetry (DSC) measurements were taken with a TA DSC 2010 apparatus (TA Instruments, New Castle, DE) with a temperature range of -50 to $+220^{\circ} \mathrm{C}$. The glass transition temperatures $\left(T_{\mathrm{g}}\right)$ were determined at a heating rate of $20^{\circ} \mathrm{C} / \mathrm{min}$. The instrument was calibrated with high-purity indium and gallium. The crystallinity of the polymers was calculated using Eq. 1 , where 퐶 (\%) is the percentage crystallinity, $\Delta H_{\mathrm{m}}^{\mathrm{m}}$ is the measured melting enthalpy $(\mathrm{J} / \mathrm{g})$ and $\Delta H_{\mathrm{m}}^{100 \%}$ is the melting enthalpy for fully crystalline polyhydroxybutyrate (PHB) (146 J/g) (Radecka et al. 2016).

$C(\%)=\frac{\Delta H_{\mathrm{m}}^{\mathrm{m}}}{\Delta H_{\mathrm{m}}^{100 \%}}$

\section{Determination of pathogenicity}

Pathogenicity assays were carried out on both B. thailandensis E264 wild-type and triple mutant strains. Analyses were carried out using the Galleria mellonella model as previously described (Irorere et al. 2018). G. mellonella were purchased from Pets at Home (Coleraine, Northern Ireland, UK), and 10 larvae were used per strain, in triplicate. Each larva was injected with $100 \mathrm{cfu}$ of each strain in $20 \mu \mathrm{l}$ of phosphate-buffered saline (PBS), with sterile PBS used as a negative control. Larvae were incubated for $48 \mathrm{~h}$ at $37{ }^{\circ} \mathrm{C}$ in perforated petri dishes. 


\section{Statistical analysis}

GraphPad Prism version 6 was used to analyse results of this study. Unless stated otherwise, analyses were carried out using one- or two-way ANOVA with Tukey's multiple comparison $(P \leq 0.05)$.

\section{Results}

\section{Effect of QS mutations on cell growth}

To determine the effect of QS mutations on B. thailandensis, strains were grown in nutrient broth supplemented with $4 \%$ glycerol at $30^{\circ} \mathrm{C}$ and $200 \mathrm{rpm}$ for $240 \mathrm{~h}$. During this period, growth was assessed by measuring the absorbance at $\mathrm{OD}_{600}$. The initial average cell concentration at the start of fermentation was approximately $\mathrm{OD}_{600}$ of 0.23 . Single mutant strains (S $\Delta$ btall, $\mathrm{S} \Delta b t a I 2$ and $\mathrm{S} \Delta b t a I 3$ ) grew to an $\mathrm{OD}_{600}$ of 8.89 , while double mutant strains (D $\Delta$ btaI2 $\Delta b t a I 3)$ grew to an $\mathrm{OD}_{600}$ of 9.22 with a maximum $\mathrm{OD}_{600}$ of 5.08 recorded for the triple mutant strain (T $\Delta$ btaIl $\Delta b t a I 2 \Delta b t a I 3)$ (Fig. S2a). Tukey multiple comparison in two-way ANOVA showed no significant difference in the growth of all single mutant strains compared with the wild type throughout the fermentation period. However, the cell concentration of the double mutant strain was significantly higher $(P<0.05)$ from $72 \mathrm{~h}$ compared with the wild type, while the cell concentration of the triple mutant was significantly lower $(P<0.05)$ from $48 \mathrm{~h}$ of fermentation compared with both the wild-type and the single mutant strains. Additionally, the triple mutant strain reached stationary phase at $24 \mathrm{~h}$ of growth, while the wild-type and other mutants reached stationary phase between $72 \mathrm{~h}$ and $96 \mathrm{~h}$ of growth (Fig. S2a).

The triple mutant strain was subsequently selected for complementation analysis, as it showed the most significant difference in cell growth when compared with the wild type. The initial complementation study was carried out in fresh nutrient broth supplemented with $40 \%$ filter-sterilised spent media from an overnight culture of wild-type $B$. thailandensis and $4 \%$ glycerol. Results showed that the triple mutant strain had similar growth patterns and microbial concentration throughout the fermentation, compared with the wild-type strain used as a control (Fig. S2b). The cell concentration of the triple mutant reached $\mathrm{OD}_{600}$ of 7.70 , similar to levels observed in wild-type cultures with or without spent medium supplementation $\left(\mathrm{OD}_{600} 8.22\right.$ and 7.02 respectively) (Fig. S2a-b).

Based on the above result, a further complementation study was designed using nutrient broth supplemented with $4 \mu \mathrm{M}$ each of the three QS signal molecules of B. thailandensis E264 (3OHC $10-\mathrm{HSL}, 3 \mathrm{OHC}_{8}$-HSL and $\mathrm{C}_{8}$-HSL). Cell growth of both the wild-type and the triple mutants in supplemented media was analysed, and results were compared with those obtained in non-supplemented media (Fig. 1a). Similar to results observed with the initial complementation study, the growth curve of the triple mutant strain was restored to patterns similar to that of the wild type after medium supplementation with synthetic QS signalling molecules. Furthermore, the cell concentration of the triple mutant $\left(\mathrm{OD}_{600}\right.$ of 9.80$)$ after $240 \mathrm{~h}$ of fermentation was at levels comparable to media supplemented $\left(\mathrm{OD}_{600}\right.$ of 9.22) and non-supplemented $\left(\mathrm{OD}_{600}\right.$ of 8.22) wild-type cultures (Fig. 1a).

Due to the significantly lower $\mathrm{OD}_{600}$ recorded for the triple mutant strain compared with the wild-type and other mutants, viable cell count was carried out for both the triple mutant strain, wild type strain and triple mutant strain in media complemented with synthetic QS signal molecules. In noncomplemented media of the triple mutant strain, the maximum number of viable cells recorded was $\log _{10} 9.97 \mathrm{cfu} / \mathrm{l}$ at $96 \mathrm{~h}$ of fermentation. After this, the cells went into death phase with a final viable cell number of $9.14 \mathrm{cfu} / \mathrm{ml}$ recorded at the end of the fermentation $(240 \mathrm{~h})$. Neither the wild-type nor triple mutant strain in complemented media went into death phase; they both maintained the stationary phase from $48 \mathrm{~h}$ and had viable cell numbers of $\log _{10} 10.04 \mathrm{CFU} / \mathrm{ml}$ and $\log _{10} 10.07 \mathrm{cfu} / \mathrm{ml}$ respectively at the end of fermentation (Fig. 1b). Additionally, the viable count at $24 \mathrm{~h}$ in the triple mutant strain was observed to be significantly lower than the wild-type strain, although the difference at $48 \mathrm{~h}$ and $96 \mathrm{~h}$ were not significant compared with either the wild-type or medium-complemented triple mutants (Fig. 1b).

\section{Glycerol consumption}

To see if the growth limitation observed in the triple mutant strain is due to their inability to utilize available nutrient, we measured the change in glycerol concentration throughout the fermentation period in all mutants' strains and the wild type. Surprisingly, we observed that glycerol concentration was consistently lower in the triple mutant compared with the WT or other mutant strains used in this study during the stationary growth phase (Fig. 2a). However, the glycerol concentrations of all the single and double mutant strains were not significantly $(P \geq 0.05)$ different from the wild type at $240 \mathrm{~h}$ of fermentation (Fig. 2a). The mean glycerol concentration of the triple mutant at $240 \mathrm{~h}$ was $8.76 \mathrm{~g} / \mathrm{l}$, which was significantly lower $(P<0.05)$ than the concentration of the wild type at $16.19 \mathrm{~g} / 1$ and other mutants at concentrations between 18.45 and $19.64 \mathrm{~g} / \mathrm{l}$ (Fig. 2a). This trend was observed from $96 \mathrm{~h}$ of growth, with the triple mutant consistently showing lower concentration of glycerol compared with either the wild-type or other mutant strains. When the triple mutant strains were grown in media supplemented with QS signal molecules, the glycerol consumption was observed 


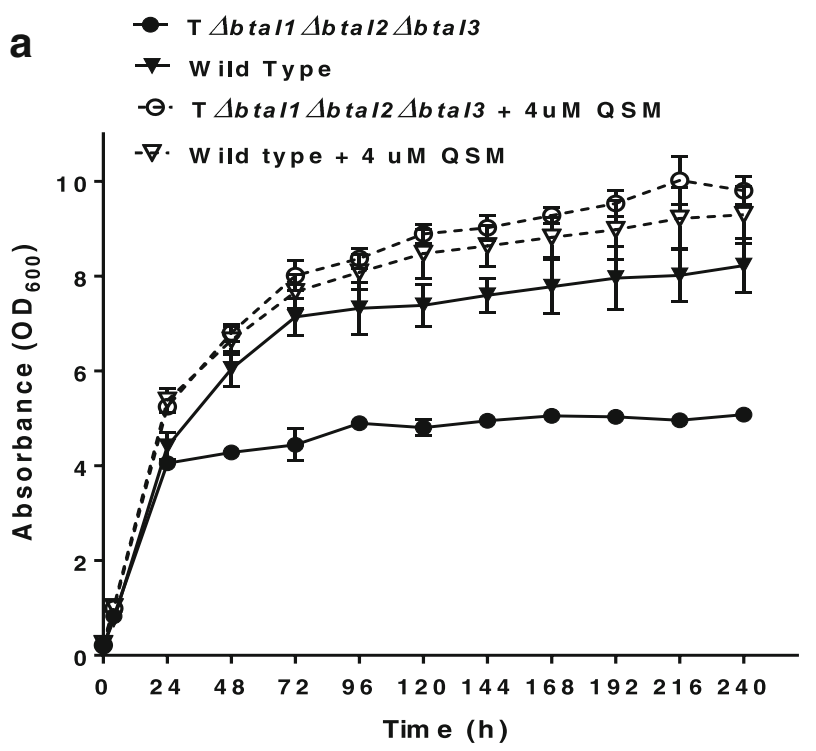

Fig. 1 Quorum sensing triple mutant experience reduced growth and death phases compared with the wild type. Growth of wild-type and triple mutant strains measured by either optical density (a) or viable cell count (b) in non-supplemented nutrient media or nutrient media supplemented

to be similar to that of the wild type with a final concentration of $18.2 \mathrm{~g} / \mathrm{l}$ at $240 \mathrm{~h}$ (Fig. 2b).

\section{Surface tension analysis and rhamnolipid production}

As an indication of biosurfactant production, the media supernatants were collected at time intervals to observe reduction in

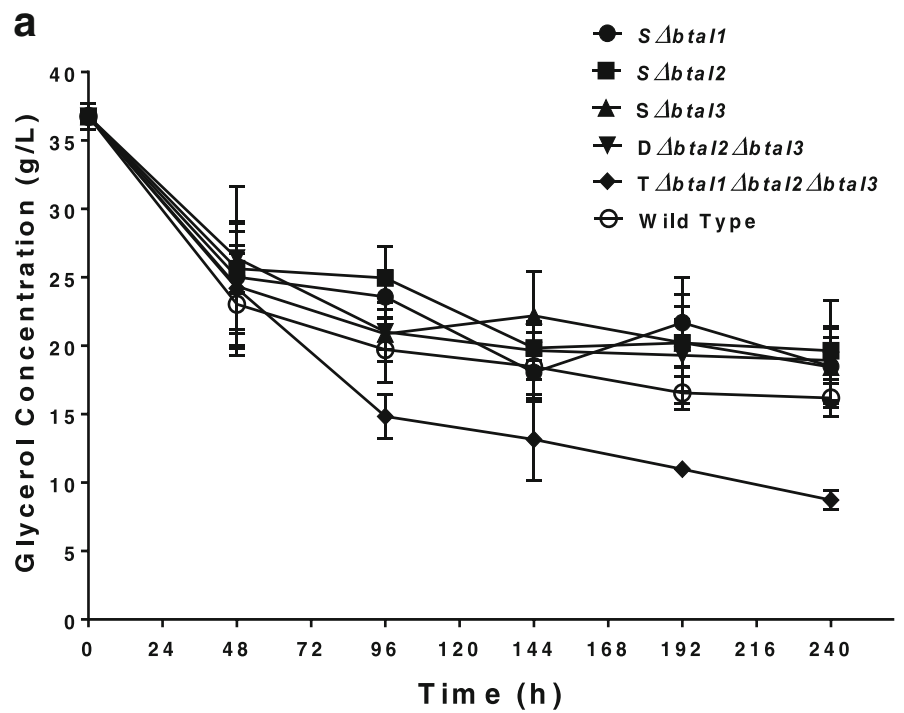

Fig. 2 There is increased glycerol metabolism by quorum sensing triple mutant strain compared with wild type or other quorum sensing mutants. a Glycerol concentration of wild-type $B$. thailandensis and all quorum sensing mutants in non-supplemented nutrient broth. b Glycerol concentration of triple mutant strain in non-supplemented nutrient broth and nutrient media supplemented with $4 \mu \mathrm{M}$ each of $3 \mathrm{OHC}_{10} \mathrm{HSL}$, $3 \mathrm{OHC}_{8} \mathrm{HSL}$ and $\mathrm{C}_{8} \mathrm{HSL}$, in comparison to wild type. Glycerol

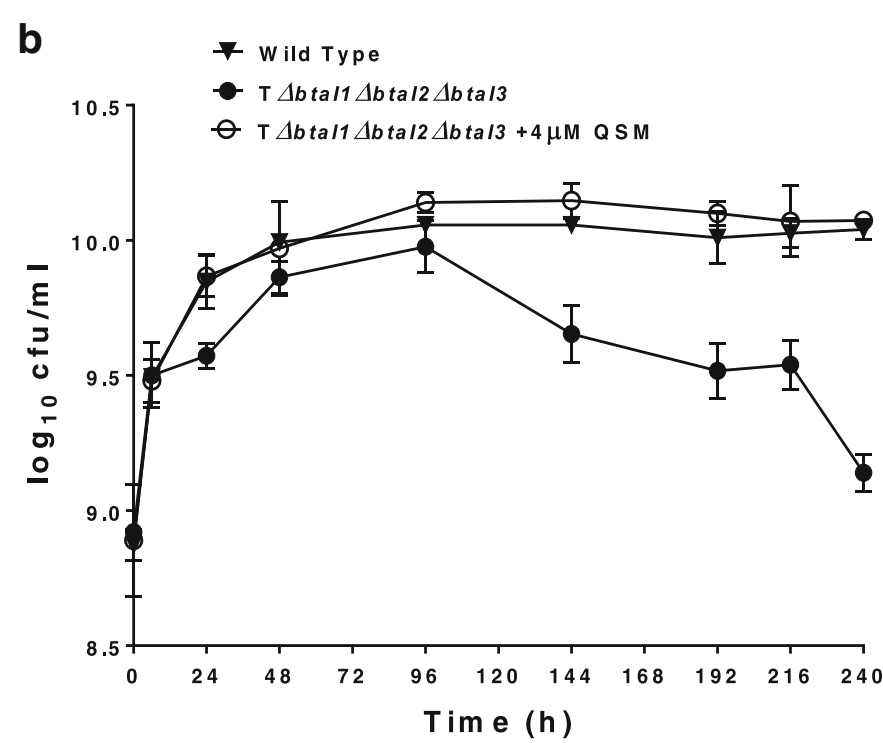

with $4 \mu \mathrm{M}$ each of $3 \mathrm{OHC}_{10} \mathrm{HSL}, 3 \mathrm{OHC}_{8} \mathrm{HSL}$ and $\mathrm{C}_{8} \mathrm{HSL}$. Viable cell numbers in cfu/ $/ \mathrm{ml}$ were converted to $\log _{10}$ values, which is represented in the $Y$-axis as $\log _{10} \mathrm{cfu} / \mathrm{ml}$. All experiments were carried out in triplicate $(n=3)$

surface tension. All single and the double mutant strains were able to reduce the surface tension of the production medium from $65.0 \mathrm{mN} / \mathrm{m}$ to approximately $41.0 \mathrm{mN} / \mathrm{m}$ at $240 \mathrm{~h}$ of fermentation, which is similar to that of the wild type (Fig. 3a). However, rather surprisingly, the triple mutant strain reduced the surface tension of the fermentation medium from 65 to $34.1 \mathrm{mN} / \mathrm{m}$ at $240 \mathrm{~h}$ of fermentation (Fig. 3a).

\section{$\mathrm{T} \Delta b t a / 1 \Delta b t a / 2 \Delta b t a / 3$}

b wild Type

T $\Delta b$ tal1 $\Delta b t a l 2 \Delta b t a l 3+4$ u M S M

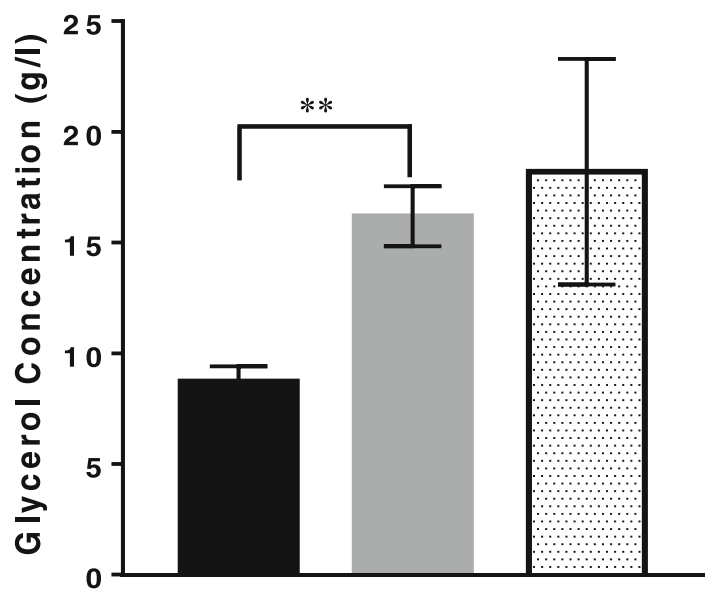

concentrations in media supernatants were assessed using a spectrophotometric method described previously (Bondioli and Della Bella 2005), and all experiments were conducted in triplicate $(n=3)$. Differences in glycerol concentration between the wild type and the triple mutant were analysed using unpaired $t$ test in GraphPad Prism 7. WT = wild type and $\mathrm{TM}=$ triple mutant 

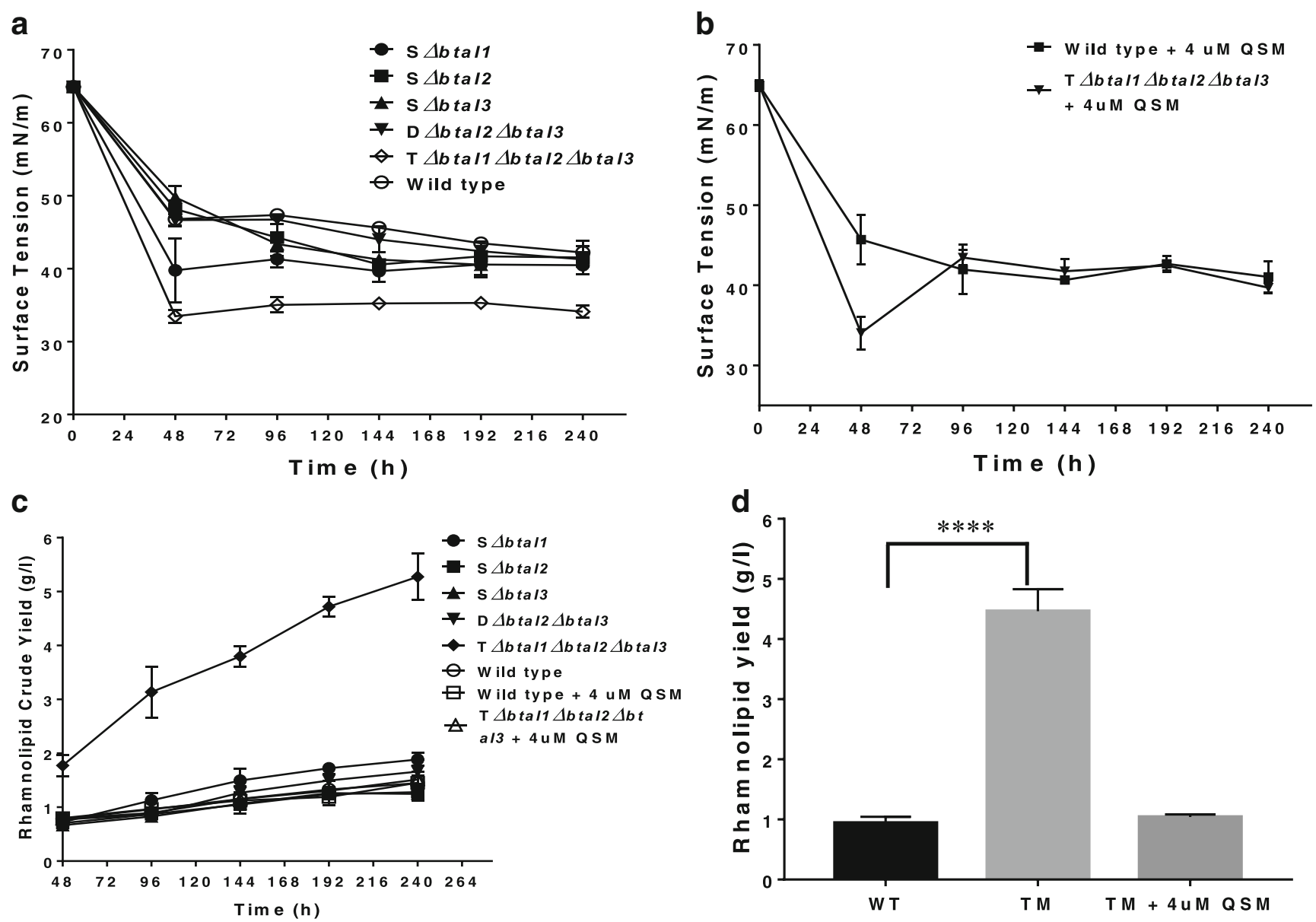

Fig. 3 Rhamnolipid production by quorum sensing triple mutant strain of $B$. thailandensis is significantly higher compared with the wild type or other mutant strains. a Surface tension of the different quorum sensing mutants of $B$. thailandensis and the wild type, showing lower surface tension of the triple mutant media compared with other strains. b Restoration of surface tension of $B$. thailandensis triple mutant media to levels comparable to the wild type when nutrient broth is supplemented with $4 \mu \mathrm{M}$ each of $3 \mathrm{OHC}_{10} \mathrm{HSL}, 3 \mathrm{OHC}_{8} \mathrm{HSL}$ and $\mathrm{C}_{8} \mathrm{HSL}$. c Crude rhamnolipid yield of all quorum sensing mutants of $B$. thailandensis

Complementation analysis in nutrient broth supplemented with $40 \%$ spent media was observed to restore the surface tension of the triple mutant to levels similar to those of the wild type grown in both $40 \%$ spent media supplemented and non-supplemented nutrient broth (Fig. S3a). This restoration was also observed in nutrient broth supplemented with $4 \mu \mathrm{M}$ each of the three QS signal molecules (Fig. 3b). However, in the latter, the surface tension at $48 \mathrm{~h}$ was lower than that of the wild type (supplemented and non-supplemented) and comparable to the triple mutant strain grown in non-supplemented media (Fig. 3b).

Following surface tension analysis, rhamnolipid extraction was carried out at different stages of fermentation and yields were compared with those obtained from the wild type (Fig. $3 \mathrm{c})$. At $48 \mathrm{~h}$ of fermentation, rhamnolipid crude yields in the three single ( $\mathrm{S} \Delta b t a I 1, \mathrm{~S} \Delta b t a I 2$ and $\mathrm{S} \Delta b t a I 3)$ and double and the wild type, showing a significantly higher yield of the triple mutant compared with other strains including the wild type. d Rhamnolipid yield of quorum sensing triple mutant grown in nonsupplemented and synthetic HSLs supplemented nutrient broth at $240 \mathrm{~h}$ compared with wild type. All experiments were carried out in triplicates $(n=3)$, and the differences in rhamnolipid yield between the wild type and the triple mutant were analysed using the unpaired $t$ test in GraphPad Prism 7

(D $\Delta$ btaI2 $\Delta$ btal3) mutant strains at $0.72 \mathrm{~g} / 1,0.70 \mathrm{~g} / 1,0.67 \mathrm{~g} /$ 1 and $0.77 \mathrm{~g} / 1$ respectively were comparable to that of the wild type at $0.79 \mathrm{~g} / \mathrm{l}$. This trend continued throughout the growth cycle ( $96 \mathrm{~h}, 144 \mathrm{~h}, 192 \mathrm{~h}$ and $240 \mathrm{~h}$ ), with no significant ( $P \geq$ $0.05)$ differences observed in the crude yield of the single and double mutants compared with the wild type (Fig. 3c). Unexpectedly, we observed that the rhamnolipid crude yield from the triple mutant (T $\Delta$ btaIl $\Delta$ btaI2 $\Delta$ btaI3) was significantly higher $(P<0.05)$ than that of the wild type and all other mutant strains. At $240 \mathrm{~h}$ of fermentation, the rhamnolipid crude yield of the triple mutant was $5.27 \pm 0.25 \mathrm{~g} / \mathrm{l}$, while that of the wild type at the same time was $1.45 \pm 0.12 \mathrm{~g} / \mathrm{l}$ (Fig. 3c). Complementation of the fermentation broth with $40 \%$ spent culture from the wild-type strain led to a reduction in the rhamnolipid crude yield of the triple mutant to $1.16 \pm 0.08 \mathrm{~g} /$ 1 at $240 \mathrm{~h}$ of fermentation (Fig. S2b). Similarly, 
complementation with $4 \mu \mathrm{M}$ each of the three different QS signal molecules, resulted in a reduction of rhamnolipid crude yield to comparable levels as the wild type (with or without QS signal molecule complementation) throughout the fermentation, with a final crude yield of $1.51 \pm 0.05 \mathrm{~g} / \mathrm{l}$ at $240 \mathrm{~h}$ (Fig. 3c).

To be sure that the high rhamnolipid yield observed in gravimetric analysis of rhamnolipid crude yield is not due to high fatty acid content in the extract, further characterisation of rhamnolipid yield at $240 \mathrm{~h}$ was carried out by LC/MS. Results showed yields of $0.94 \pm 0.06 \mathrm{~g} / 1,1.04 \pm 0.03 \mathrm{~g} / \mathrm{l}$ and $4.46 \pm 0.345 \mathrm{~g} / \mathrm{l}$ for the wild type, medium-complemented triple mutant and the triple mutant in non-complemented media respectively (Fig. 3d).

To see if the increase in rhamnolipid production had any effect on the pathogenicity of B. thailandensis, the Galleria mellonella model of pathogenicity assessment was used to compare the pathogenicity of wild type and the triple mutant strain. Larvae were infected with 100 cfu each of either wildtype or triple mutant strain. The result presented showed that both strains had up to $94 \%$ survival of the larvae at $18 \mathrm{~h}$ of infection. However, the triple mutant strains had about 33\% survival at $30 \mathrm{~h}$, lower than larvae infected with the wild-type strain, with $90 \%$ survival. However, there was a $100 \%$ mortality or $0 \%$ survival after $48 \mathrm{~h}$ of infection with either strain (Fig. S3c).

\section{Rhamnolipid characterisation}

The purified rhamnolipid from the wild type and the triple mutant strain were analysed by LC/MS, and the relative proportions of the various congeners in both strains were compared. Characterisation was carried out on purified rhamnolipid from independent triplicate experiment, and results are summarised in Table 2, with similar congeners observed in both strains. As expected, the dirhamnolipids were the most abundant in the wild-type strain compared with the monorhamnolipids $(67.5 \pm 5.3$ to $32.5 \pm 3.1$, respectively), with a lipid chain length of $\mathrm{C}_{14}-\mathrm{C}_{14}$ found to be the most abundant mono- and dirhamnolipid congeners. In contrast, we observed that monorhamnolipids were the most abundant congeners compared with dirhamnolipids ( $53.7 \pm 5.7$ to 46.3 \pm 6.3 respectively) in the triple mutant strain. However, rhamnolipids with a lipid chain length of $\mathrm{C}_{14}-\mathrm{C}_{14}$ remained the most abundant mono- and dirhamnolipids congeners, as observed in the wild-type strain.

When we supplemented the rhamnolipid production medium with QS signal molecules, results indicate a similar trend of rhamnolipids compared with triple mutants grown in supplemented media from the wild type; dirhamnolipids were observed as the most abundant congeners compared with monorhamnolipids. Additionally, the relative abundance of dirhamnolipid in the triple mutant grown in QS signal molecule supplemented media was observed to be significantly higher than that of the wild type grown in nonsupplemented media ( $86.8 \pm 7.6$ and $67.5 \pm 5.3$ respectively). However, it was similar to the wild type grown in QS signal molecule-supplemented media $(90.5 \pm 20.2)$ (Table 2$)$.

\section{PHA production and characterisation}

To investigate if increased rhamnolipid production is due to reduction in the storage of carbon as polyhydroxyalkanoate (PHA), the PHA yield by the QS complete mutant was assessed and compared with that of the wild type. PHA yield was observed to peak at $144 \mathrm{~h}$ of fermentation in both the wild type and mutant strains, with the wild-type strain consistently having a significantly higher $(P<0.05)$ PHA yield compared with the mutant strain (Fig. 4a). The highest yields observed were $1.41 \pm 0.12 \mathrm{~g} / 1$ and $0.58 \pm 0.09 \mathrm{~g} / \mathrm{l}$, from the wild type and the triple mutant strains respectively. To identify if this significantly lower yield can be accounted for by the lower growth rate observed in the triple mutant strain, the percentage yield per cell dry weight was calculated. The wild-type strain also had a consistently higher percentage yield (g/gCDW) compared with the triple mutant strain, with the highest recorded percentage yield of $31.4 \%$ and $21.8 \%$ respectively at $96 \mathrm{~h}$ of fermentation (Fig. $4 \mathrm{~b}$ ).

Both ${ }^{1} \mathrm{H}$ and ${ }^{13} \mathrm{C}$ NMR confirmed that poly-3hydroxybutyrate was the primary PHA produced by both the complete mutant and the wild-type strain of $B$. thailandensis (Fig. 4c, d and Fig. S4a-b). DSC traces of PHA from wild-type and complete mutant strains showed that the glass transition temperatures were $3.2{ }^{\circ} \mathrm{C}$ and $1.2{ }^{\circ} \mathrm{C}$ respectively, with melting temperatures of $172.1{ }^{\circ} \mathrm{C}$ and $165.3{ }^{\circ} \mathrm{C}$ respectively. Additionally, the crystallinity of the polymers were $52.2 \%$ and $51.2 \%$ for wild-type and complete mutant strains respectively.

\section{Discussion}

The classification of $B$. thailandensis E264 as a biosafety level I or a putative non-pathogen makes it an attractive substitute for rhamnolipid production, compared with $P$. aeruginosa which is an opportunistic pathogen or biosafety level II organism (Brett et al. 1998; Irorere et al. 2017). However, low levels of rhamnolipid production and the significantly longer fermentation time required to produce substantial quantities of rhamnolipid are major factors affecting the implementation of B. thailandensis in the industrial production of rhamnolipid. Iron limitation in the production media and the introduction of exogenous quorum sensing molecules have been suggested as ways of inducing early rhamnolipid synthesis and improving overall rhamnolipid yield (Irorere et al. 2018). This study was designed to assess the effect of quorum sensing mutations on 

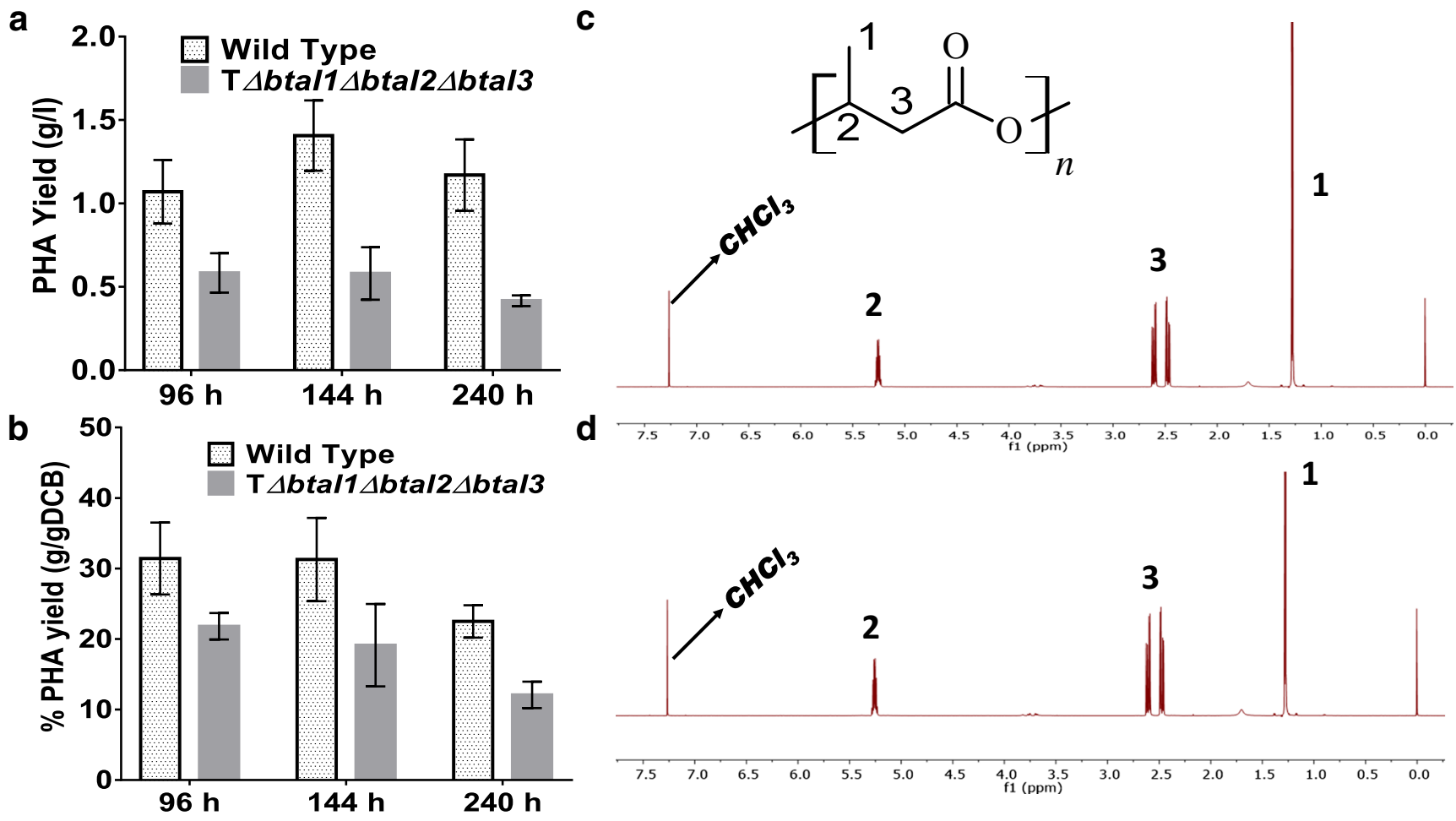

Fig. 4 Polyhydroxyalkanoate (PHA) yield is lower in B. thailandensis quorum sensing triple mutant compared with wild type. a, b PHA yield $(\mathrm{g} / \mathrm{l})$ and percentage $\mathrm{PHA}$ productivity $(\mathrm{g} / \mathrm{gDCB})$ respectively at different stages of fermentation of wild-type and triple mutant strains of B. thailandensis. $\mathbf{c}, \mathbf{d} \mathrm{H}^{\prime} \mathrm{NMR}$ of polyhydroxybutyrate (PHB) produced

by wild-type and triple mutant strains of $B$. thailandensis E264 respectively. PHA was extracted from lyophilised biomass by solvent extraction with chloroform followed by precipitation with ice-cold methanol. Results presented are biological triplicates $(n=3)$

rhamnolipid production by $B$. thailandensis E264 and subsequently to determine the effect, if any, of exogenous addition of quorum sensing molecules on rhamnolipid production by B. thailandensis.

$B$. thailandensis is known to have three different quorum sensing circuits, BtaI1-BtaR1, BtaI2-BtaR2 and BtaI3BtaR3, that respond to or direct the synthesis of $\mathrm{C}_{8}$-HSLs, $3 \mathrm{OHC}_{10}$-HSLs and $3 \mathrm{OHC}_{8}$-HSLs (Chandler et al. 2009; Le Guillouzer et al. 2017), with two orphan LuxR signal receptors, lacking the known LuxI signal synthase, BtaR4 and BtaR5. Mutations in any or all of the LuxI signal synthase will result in the cell's inability to synthesize the corresponding signal molecules. Subsequently, the corresponding LuxR signal receptors are orphaned and unable to direct the synthesis of the particular signal molecule, unless there is the addition of respective exogenous signal molecules. Based on this, we assessed the rhamnolipid production of either single (S $\Delta b t a I 1, \mathrm{~S} \Delta b t a I 2$ and $\mathrm{S} \Delta b t a I 3)$, double (D $\Delta b t a I 2 \Delta b t a I 3$ ) or triple (T $\Delta$ btaI1 $\Delta$ btaI2 $\Delta$ btaI3) mutant strains of B. thailandensis E264 to see the effect of quenching single or multiple QS circuits on rhamnolipid synthesis in B. thailandensis E264.
While assessing the growth of $B$. thailandensis during rhamnolipid production, we observed that the triple mutant strain reached stationary phase earlier and at significantly lower cell density compared with both the wild type and the double or all three single mutant strains (Fig. S1a and Fig. 1a). This was a surprising and unexpected discovery as QS mutations or quorum quenching (QQ) in most microbial strains including Vibrio harveyi, Yersinia ruckeri, Sodalis praecaptivus, P. aeruginosa and Burkholderia glumae have been shown to either have no effect or to enhance microbial growth or growth rate under different experimental conditions (Enomoto et al. 2017; Ishida et al. 2007; Nackerdien et al. 2008; Nickzad and Déziel 2016; Torabi Delshad et al. 2018). In the above studies, QS mutants or WT strains growing in the presence of quorum quenching (QQ) molecules showed comparable or enhanced growth compared with the wild type.

One report that showed growth limitation as a consequence of QS mutation was Goo et al. (2012). In this report, mutant strains of B. glumae, B. pseudomallei and B. thailandensis deficient in $\mathrm{C}_{8}$-HSL synthesis experienced a rapid decline in cell population, due to increased alkalinity of the media, as the cells enter into late stationary phase in a nutrient-rich medium, 
compared with the WT (Goo et al. 2012). However, a more recent study did not observe any effect on growth of a $\mathrm{C}_{8}$-HSL deficient mutant of $B$. glumae (tof $l$ ) in both minimal and rich media (Nickzad and Déziel 2016). Similarly, no decline in the cell population of a $\mathrm{C}_{8}$-HSL-deficient mutant of B. thailandensis ( $\mathrm{S} \Delta$ btaIl) was observed in our study, with a sustained stationary phase observed throughout the period of fermentation (Fig. S1a).

It has previously been reported that quorum sensing mutations affect self-aggregation of $B$. thailandensis in minimal media (Chandler et al. 2009). To ensure that the lower $\mathrm{OD}_{600}$ observed in the triple mutant is not a result of differences in self-aggregation in the cultures, viable cell count was carried out. Results showed that cell count of the triple mutant was significantly lower at $24 \mathrm{~h}$. However, the differences in cell count compared with the wild type and the triple mutant growing in the presence of HSLs were not significant at $48 \mathrm{~h}$ and $96 \mathrm{~h}$, with the triple mutant strain going into death phase after $96 \mathrm{~h}$ (Fig. 1b). The fact that the death phase observed during viable cell count is not seen in the OD reading suggests the potential of increased accumulation of cell exudates in the media, including rhamnolipid; this may also explain the decline in cell number due to cell toxicity.

However, it is also possible that the decline in cell number is due to the inability of the triple mutant to utilise available substrates as previously reported in other microbial strains including P. aeruginosa (Antunes et al. 2007; Pearson et al. 1997; Studer et al. 2008). To see if this is true for B. thailandensis, we studied the change in glycerol concentration throughout the fermentation period. Surprisingly, we observed that glycerol concentration was consistently lower in the triple mutant compared with the wild-type or other mutant strains used in this study during stationary growth phase (Fig. 2a). Both growth and glycerol utilisation were restored to comparable levels as the wild type when triple mutant strains are grown in media supplemented with wild-type spent media or $4 \mu \mathrm{M}$ of each of the $3 \mathrm{QS}$ signal molecules. This implies that glycerol was utilised much faster in the triple mutant compared with both the wild-type and other mutant strains. This result is in agreement with a previous study in which QS was shown to have no effect on the metabolic diversity of B. thailandensis as it does in P. aeruginosa (Chandler et al. 2009). Indeed, QS was reported to negatively influence the rate of respiration of $B$. thailandensis on most carbon, nitrogen and phosphorus sources (Chandler et al. 2009). Collectively, these results suggest that growth limitation observed in QS triple mutant is not due to their inability to utilize available carbon or energy sources.

As QS is known to control the gene expression of a wide range of secondary metabolites in B. thailandensis (Majerczyk et al. 2014), one possible explanation for growth limitation could be the overexpression and/or continuous activation of 'unnecessary' genes, thus resulting in increased demand on the cell's energy supply, thus trading off growth for continuous production of metabolites. This may also explain the increased glycerol metabolism by the cell. As to why this effect is not observed in single or double mutant strains, reports have shown an overlap in genes regulated by the different QS circuits (Majerczyk et al. 2014). Thus, some of the effect of mutations in one QS circuit can be compensated for by another.

Rhamnolipid extraction and quantification showed that the triple mutant had a significantly higher crude rhamnolipid yield compared with both the wild type and other mutant strains, while the differences in crude yield of the single and double mutants were not significant compared with the wild type (Fig. 3c). Thus, only the wild type and the triple mutant strain were subsequently selected for rhamnolipid quantification by HPLC-MS. Data obtained from LC-MS showed that the QS triple mutant produced over 4 times more rhamnolipid compared with the wild type, with complementation restoring rhamnolipid production to similar levels as the wild type (Fig. $3 \mathrm{~d})$. This therefore confirms that rhamnolipid production is significantly higher in triple mutant compared with the wild type.

We also wanted to know if the increase in glycerol consumption led to the accumulation of more energy storage by the cell in the form of PHA. To do this, PHAs were extracted and quantified from both the wild-type and triple mutant strains. This was also carried out because previous studies on $P$. aeruginosa have shown that QS mutants have significantly lower levels of PHA production (Xu et al. 2011). We have also previously shown that PHA mutant strains of $B$. thailandensis produce significantly higher quantities of rhamnolipid compared with WT (Funston et al. 2017). Hence, it is possible that carbon is pulled away from energy storage towards rhamnolipid biosynthesis. We therefore hypothesise that the increased rhamnolipid production is in part due to a reduction in PHA biosynthesis, increased glycerol metabolism and reduced growth.

As expected, PHA production was consistently higher in the WT compared with the triple mutant at different stages of extraction (Fig. 4a). To be sure that this higher PHA yield is not due to reduction in cell biomass, we calculated the percentage PHA per cell ( $\mathrm{g} / \mathrm{gCDB})$. Results showed that the percentage PHA (g/gDCB) of the triple mutant was also consistently lower than the WT (Fig. 4b). QS does not, however, have any effect on the structural properties of PHA produced by $B$. thailandensis in this study, with PHB identified as the main product in both strains (Fig. 4c, d). However, DSC characterisation showed reductions in the glass transition and melting temperatures of PHA from triple mutant compared with the wild-type strain. The thermal properties of PHA from both strains were however consistent with those of PHB reported previously (Irorere et al. 2014; Radecka et al. 2016). 
Table 1 Average relative abundance of purified rhamnolipid congeners produced by wild-type and triple mutant of B. thailandensis E264. Results presented are averages of analysis from biological triplicates of rhamnolipid extracts, and the error values are indicative of the standard deviation of the mean. $\mathrm{ND}=$ below the level of detection

\begin{tabular}{|c|c|c|c|c|}
\hline \multirow[t]{2}{*}{ Rhamnolipid congener } & \multirow[t]{2}{*}{ Pseudomolecular ion $(\mathrm{m} / \mathrm{z})$} & \multirow[t]{2}{*}{ Retention time (min) } & \multicolumn{2}{|c|}{ Relative abundance (\%) } \\
\hline & & & Wild type & Triple mutant \\
\hline Rha $-\mathrm{C}_{10}-\mathrm{C}_{12} / \mathrm{C}_{12}-\mathrm{C}_{10}$ & 531 & 23.17 & ND & $1.31 \pm 0.14$ \\
\hline Rha $-\mathrm{C}_{12}-\mathrm{C}_{12} / \mathrm{C}_{10}-\mathrm{C}_{14} / \mathrm{C}_{14}-\mathrm{C}_{10}$ & 559 & 25.58 & $1.65 \pm 0.27$ & $6.42 \pm 0.78$ \\
\hline Rha $-\mathrm{C}_{12}-\mathrm{C}_{14} / \mathrm{C}_{14}-\mathrm{C}_{12}$ & 587 & 27.72 & $5.35 \pm 0.49$ & $18.01 \pm 1.86$ \\
\hline Rha $-\mathrm{C}_{14}-\mathrm{C}_{14}$ & 615 & 29.32 & $23.65 \pm 1.62$ & $26.88 \pm 2.84$ \\
\hline Rha $-\mathrm{C}_{14}-\mathrm{C}_{16} / \mathrm{C}_{16}-\mathrm{C}_{14}$ & 643 & 30.73 & $1.85 \pm 0.67$ & $1.04 \pm 0.11$ \\
\hline Total monorhamnolipid & & & $32.50 \pm 3.05$ & $53.66 \pm 5.73$ \\
\hline Rha-Rha- $\mathrm{C}_{10}-\mathrm{C}_{12} / \mathrm{C}_{12}-\mathrm{C}_{10}$ & 677 & 19.16 & $1.35 \pm 0.37$ & $2.2 \pm 0.43$ \\
\hline Rha-Rha- $\mathrm{C}_{12}-\mathrm{C}_{12} / \mathrm{C}_{10}-\mathrm{C}_{14} / \mathrm{C}_{14}-\mathrm{C}_{10}$ & 705 & 22.81 & $8.69 \pm 1.56$ & $11.17 \pm 2.59$ \\
\hline Rha-Rha- $\mathrm{C}_{12}-\mathrm{C}_{14} / \mathrm{C}_{14}-\mathrm{C}_{12}$ & 733 & 25.19 & $15.32 \pm 0.96$ & $15.62 \pm 2.14$ \\
\hline Rha-Rha- $\mathrm{C}_{14}-\mathrm{C}_{14}$ & 761 & 27.21 & $36.99 \pm 1.91$ & $16.03 \pm 0.94$ \\
\hline Rha-Rha- $\mathrm{C}_{14}-\mathrm{C}_{16} / \mathrm{C}_{16}-\mathrm{C}_{14}$ & 789 & 28.94 & $5.16 \pm 0.48$ & $1.32 \pm 0.23$ \\
\hline Total dirhamnolipid & & & $67.51 \pm 5.28$ & $46.34 \pm 6.33$ \\
\hline
\end{tabular}

As rhamnolipid is a known virulence factor in many Gramnegative bacterial strains, including $P$. aeruginosa, B. glumae and B. pseudomallei (Häußler et al. 2003; Nickzad et al. 2015; Zulianello et al. 2006), we hypothesise that the increased rhamnolipid yield in the triple mutant strain might also increase their virulence. Using the G. mellonella model of pathogenicity, we observed a similar pattern in the mortality of $G$. mellonella by both triple mutant and WT strains. However, we observed a higher mortality at $30 \mathrm{~h}$ of larval inoculation after infection with the triple mutant compared with the WT. However, this is not comparable to the pathogenicity of $P$. aeruginosa which showed a $100 \%$ mortality of $G$. mellonella larvae at $18 \mathrm{~h}$ of inoculation under similar experimental conditions (Irorere et al. 2018).

Table 2 Average relative abundance of purified rhamnolipid congeners produced by wild-type and triple mutants of $B$. thailandensis E264 grown in the presence of $4 \mu \mathrm{M}$ each $3 \mathrm{OHC}_{10} \mathrm{HSL}, 3 \mathrm{OHC}_{8} \mathrm{HSL}$ and $\mathrm{C}_{8} \mathrm{HSL}$.
Indeed, an earlier study has shown that QS is not a virulence determinant in B. thailandensis, as it is in the closely related $B$. pseudomallei, using the mouse model (Chandler et al. 2009). In their study, Chandler et al. did not observe any significant difference in the pathogenicity of triple mutant $B$. thailandensis strain on mice compared with the WT (Chandler et al. 2009). Together, these results suggest that QS has a negligible effect on B. thailandensis virulence. This is contrary to results reported in other bacterial strains including the closely related B. pseudomallei, where QS mutations are known to attenuate virulence (Ulrich et al. 2004). Hence, the negligible increase in pathogenicity observed in B. thailandensis further supports the fact that QS is a

Results presented are averages of analysis from biological triplicates of rhamnolipid extracts, and the error values are indicative of the standard deviation of the mean. $\mathrm{ND}=$ below the level of detection

\begin{tabular}{|c|c|c|c|c|}
\hline \multirow[t]{2}{*}{ Rhamnolipid congener } & \multirow[t]{2}{*}{ Pseudomolecular ion $(\mathrm{m} / \mathrm{z})$} & \multirow[t]{2}{*}{ Retention time (min) } & \multicolumn{2}{|c|}{ Relative abundance (\%) } \\
\hline & & & Wild type & Triple mutant \\
\hline Rha $-\mathrm{C}_{12}-\mathrm{C}_{14} / \mathrm{C}_{14}-\mathrm{C}_{12}$ & 587 & 27.72 & $1.49 \pm 0.50$ & $1.77 \pm 0.51$ \\
\hline Rha- $\mathrm{C}_{14}-\mathrm{C}_{14}$ & 615 & 29.32 & $7.49 \pm 4.1$ & $10.97 \pm 2.68$ \\
\hline Rha $-\mathrm{C}_{14}-\mathrm{C}_{16} / \mathrm{C}_{16}-\mathrm{C}_{14}$ & 643 & 30.73 & $0.52 \pm 0.23$ & $0.51 \pm 0.17$ \\
\hline Total monorhamnolipid & & & $9.50 \pm 4.85$ & $13.25 \pm 3.36$ \\
\hline Rha-Rha- $\mathrm{C}_{10}-\mathrm{C}_{12} / \mathrm{C}_{12}-\mathrm{C}_{10}$ & 677 & 19.16 & $1.91 \pm 0.76$ & $2.16 \pm 0.47$ \\
\hline Rha-Rha- $\mathrm{C}_{12}-\mathrm{C}_{12} / \mathrm{C}_{10}-\mathrm{C}_{14} / \mathrm{C}_{14}-\mathrm{C}_{10}$ & 705 & 22.81 & $12.72 \pm 6.74$ & $11.31 \pm 3.76$ \\
\hline Rha-Rha- $\mathrm{C}_{12}-\mathrm{C}_{14} / \mathrm{C}_{14}-\mathrm{C}_{12}$ & 733 & 25.19 & $22.29 \pm 4.59$ & $17.39 \pm 1.23$ \\
\hline Rha-Rha- $\mathrm{C}_{14}-\mathrm{C}_{14}$ & 761 & 27.21 & $49.68 \pm 6.18$ & $50.61 \pm 1.15$ \\
\hline Rha-Rha- $\mathrm{C}_{14}-\mathrm{C}_{16} / \mathrm{C}_{16}-\mathrm{C}_{14}$ & 789 & 28.94 & $3.90 \pm 1.92$ & $5.3 \pm 1.03$ \\
\hline Total dirhamnolipid & & & $90.5 \pm 20.19$ & $86.77 \pm 7.64$ \\
\hline
\end{tabular}


negative regulator of rhamnolipid production in B. thailandensis compared with other microbial strains discussed earlier.

Purified rhamnolipids from both triple mutant and WT were characterised by LC/MS. As expected, dirhamnolipids were the most predominant congeners in WT B. thailandensis. However, monorhamnolipids were observed as the most predominant congeners in the triple mutant strain (Table 1). These results are an average of rhamnolipid from biological triplicates to ensure that relative abundances are not due to experimental errors. Complementation of the media with synthetic HSLs in the triple mutant strain led to the restoration of dirhamnolipids as the predominant rhamnolipid congeners (Table 2), thus confirming that QS does indeed affect rhamnolipid congener distribution in B. thailandensis, favouring the production of dirhamnolipids. The increased abundance of monorhamnolipids in the triple mutant compared with the wild type may account for the lower surface tension observed in the former compared with the latter (Fig. 3a). This is because monorhamnolipids are known to reduce surface tension to values lower than dirhamnolipids (Dubeau et al. 2009; Tiso et al. 2017).

Additionally, we observed a significantly higher dirhamnolipid proportion in the wild-type strain grown in the presence of exogenous HSLs compared with the wildtype strain grown without exogenous HSLs. To the best of our knowledge, this is the first report implicating rhamnolipid congener distribution with QS. However, no significant difference was observed in the relative abundance of dirhamnolipid in wild-type and triple mutant strains grown in the presence of exogenous HSLs.

In conclusion, our report has shown that triple QS mutation in B. thailandensis produced higher rhamnolipid yield compared with the wild type, partly by increased metabolism and reduced accumulation of storage material in the form of PHA. Additionally, QS was also shown to affect rhamnolipid congener distribution by favouring the synthesis of dirhamnolipids over monorhamnolipids. Furthermore, introduction of acyl-HSLs in production media increased the relative abundance of dirhamnolipids compared with monorhamnolipids. Thus, QS and acy-HSLs could be a way to direct the synthesis of desired rhamnolipid congeners in B. thailandensis.

Acknowledgements The authors would like to thank Sarah Dobbin for her technical support during the analysis of rhamnolipid samples. VUI was supported by an Ulster University Vice Chancellor's Research Scholarship.

\section{Compliance with ethical standards}

Conflict of interest The authors declare that they have no conflict of interest.
Ethical statement This article does not contain any studies with human participants or vertebrates performed by any of the authors. Galleria mellonella was used for pathogenicity analysis.

Open Access This article is distributed under the terms of the Creative Commons Attribution 4.0 International License (http:// creativecommons.org/licenses/by/4.0/), which permits unrestricted use, distribution, and reproduction in any medium, provided you give appropriate credit to the original author(s) and the source, provide a link to the Creative Commons license, and indicate if changes were made.

\section{References}

Ahmed SAKS, Rudden M, Smyth TJ, Dooley JSG, Marchant R, Banat IM (2019) Natural quorum sensing inhibitors effectively downregulate gene expression of Pseudomonas aeruginosa virulence factors. Appl Microbiol Biotechnol 103(8):3521-3535. https://doi.org/10. 1007/s00253-019-09618-0

Antunes LC, Schaefer AL, Ferreira RB, Qin N, Stevens AM, Ruby EG, Greenberg EP (2007) Transcriptome analysis of the Vibrio fischeri LuxR-LuxI regulon. J Bacteriol 189(22):8387-8391

Bassler BL, Losick R (2006) Bacterially speaking. Cell 125(2):237-246. https://doi.org/10.1016/j.cell.2006.04.001

Bjarnsholt T, Jensen PO, Burmolle M, Hentzer M, Haagensen JA, Hougen HP, Calum H, Madsen KG, Moser C, Molin S, Hoiby N, Givskov M (2005) Pseudomonas aeruginosa tolerance to tobramycin, hydrogen peroxide and polymorphonuclear leukocytes is quorum-sensing dependent. Microbiology 151(2):373-383

Bondioli P, Della Bella L (2005) An alternative spectrophotometric method for the determination of free glycerol in biodiesel. Eur J Lipid Sci Technol 107(3):153-157. https://doi.org/10.1002/ejlt.200401054

Brett PJ, DeShazer D, Woods DE (1998) Burkholderia thailandensis sp nov, a Burkholderia pseudomallei-like species. Int J Syst Evol Microbiol 48(1):317-320

Chandler JR, Duerkop BA, Hinz A, West TE, Herman JP, Churchill MEA, Skerrett SJ, Greenberg EP (2009) Mutational analysis of Burkholderia thailandensis quorum sensing and self-aggregation. J Bacteriol 191(19):5901-5909. https://doi.org/10.1128/jb.00591-09

Dubeau D, Deziel E, Woods D, Lepine F (2009) Burkholderia thailandensis harbors two identical $r h l$ gene clusters responsible for the biosynthesis of rhamnolipids. BMC Microbiol 9(1):263

Duerkop BA, Varga J, Chandler JR, Peterson SB, Herman JP, Churchill MEA, Parsek MR, Nierman WC, Greenberg EP (2009) Quorumsensing control of antibiotic synthesis in Burkholderia thailandensis. J Bacteriol 191(12):3909-3918. https://doi.org/10. 1128/jb.00200-09

Dusane DH, Zinjarde SS, Venugopalan VP, McLean RJ, Weber MM, Rahman PK (2010) Quorum sensing: implications on rhamnolipid biosurfactant production. Biotechnol Genet Eng Rev 27:159-184

Enomoto S, Chari A, Clayton AL, Dale C (2017) Quorum sensing attenuates virulence in Sodalis praecaptivus. Cell Host Microbe 21(5): 629-636

Funston SJ, Tsaousi K, Smyth TJ, Twigg MS, Marchant R, Banat IM (2017) Enhanced rhamnolipid production in Burkholderia thailandensis transposon knockout strains deficient in polyhydroxyalkanoate (PHA) synthesis. Appl Microbiol Biotechnol 101(23):8443-8454. https://doi.org/10.1007/s00253017-8540-x

Goo E, Majerczyk CD, An JH, Chandler JR, Seo Y-S, Ham H, Lim JY, Kim H, Lee B, Jang MS, Greenberg EP, Hwang I (2012) Bacterial quorum sensing, cooperativity, and anticipation of stationary-phase stress. Proc Natl Acad Sci U S A 109(48):19775-19780 
Häußler S, Rohde M, von Neuhoff N, Nimtz M, Steinmetz I (2003) Structural and functional cellular changes induced by Burkholderia pseudomallei rhamnolipid. Infect Immun 71(5): 2970-2975. https://doi.org/10.1128/iai.71.5.2970-2975.2003

Irorere VU, Bagheriasl S, Blevins M, Kwiecień I, Stamboulis A, Radecka I (2014) Electrospun fibres of polyhydroxybutyrate synthesized by Ralstonia eutropha from different carbon sources. Int J Poly Sci 2014:1-11. https://doi.org/10.1155/2014/705359

Irorere VU, Tripathi L, Marchant R, McClean S, Banat IM (2017) Microbial rhamnolipid production: a critical re-evaluation of published data and suggested future publication criteria. Appl Microbiol Biotechnol 101(10):3941-3951. https://doi.org/10.1007/s00253017-8262-0

Irorere VU, Smyth TJ, Cobice D, McClean S, Marchant R, Banat IM (2018) Fatty acid synthesis pathway provides lipid precursors for rhamnolipid biosynthesis in Burkholderia thailandensis E264. Appl Microbiol Biotechnol 102:6163-6174. https://doi.org/10.1007/ s00253-018-9059-5

Ishida T, Ikeda T, Takiguchi N, Kuroda A, Ohtake H, Kato J (2007) Inhibition of quorum sensing in Pseudomonas aeruginosa by $\mathrm{N}$-acyl cyclopentylamides. Appl Environ Microbiol 73(10):3183-3188

Le Guillouzer S, Groleau M-C, Déziel E (2017) The complex quorum sensing circuitry of Burkholderia thailandensis is both hierarchically and homeostatically organized. mBio $8(6)$. https://doi.org/10. $1101 / 128819$

Majerczyk C, Brittnacher M, Jacobs M, Armour CD, Radey M, Schneider E, Phattarasokul S, Bunt R, Greenberg EP (2014) Global analysis of the Burkholderia thailandensis quorum sensing-controlled regulon. J Bacteriol 196(7):1412-1424. https:// doi.org/10.1128/jb.01405-13

Marketon MM, Glenn SA, Eberhard A, González JE (2003) Quorum sensing controls exopolysaccharide production in Sinorhizobium meliloti. J Bacteriol 185(1):325-331. https://doi.org/10.1128/jb. 185.1.325-331.2003

McKnight SL, Iglewski BH, Pesci EC (2000) The Pseudomonas quinolone signal regulates rhl quorum sensing in Pseudomonas aeruginosa. J Bacteriol 182(10):2702-2708

Miles AA, Misra S, Irwin J (1938) The estimation of the bactericidal power of the blood. Epidemiol Infect 38(6):732-749

Nackerdien ZE, Keynan A, Bassler BL, Lederberg J, Thaler DS (2008) Quorum sensing influences Vibrio harveyi growth rates in a manner not fully accounted for by the marker effect of bioluminescence. PLoS One 3(2):e1671. https://doi.org/10.1371/journal.pone. 0001671

Nakata K, Yoshimoto A, Yamada Y (1998) Correlation between autoinducers and rhamnolipids production by Pseudomonas aeruginosa IFO 3924. J Ferment Bioeng 86(6):608-610. https:// doi.org/10.1016/S0922-338X(99)80016-6

Nickzad A, Déziel E (2016) Adaptive significance of quorum sensingdependent regulation of rhamnolipids by integration of growth rate in Burkholderia glumae: a trade-off between survival and efficiency. Front Microbiol 7(1215). https://doi.org/10.3389/fmicb.2016.01215

Nickzad A, Lépine F, Déziel E (2015) Quorum sensing controls swarming motility of Burkholderia glumae through regulation of rhamnolipids. PLoS One 10(6):e0128509. https://doi.org/10.1371/ journal.pone.0128509

Ochsner UA, Koch AK, Fiechter A, Reiser J (1994) Isolation and characterization of a regulatory gene affecting rhamnolipid biosurfactant synthesis in Pseudomonas aeruginosa. J Bacteriol 176(7):20442054

Papenfort K, Bassler BL (2016) Quorum sensing signal-response systems in gram-negative bacteria. Nat Rev Microbiol 14:576-588. https://doi.org/10.1038/nrmicro.2016.89

Pearson JP, Gray KM, Passador L, Tucker KD, Eberhard A, Iglewski BH, Greenberg EP (1994) Structure of the autoinducer required for expression of Pseudomonas aeruginosa virulence genes. Proc Natl Acad Sci U S A 91(1):197-201

Pearson JP, Passador L, Iglewski BH, Greenberg EP (1995) A second Nacylhomoserine lactone signal produced by Pseudomonas aeruginosa. Proc Natl Acad Sci U S A 92(5):1490-1494

Pearson JP, Pesci EC, Iglewski BH (1997) Roles of Pseudomonas aeruginosa las and rhl quorum-sensing systems in control of elastase and rhamnolipid biosynthesis genes. J Bacteriol 179(18):5756-5767

Pesci EC, Milbank JB, Pearson JP, McKnight S, Kende AS, Greenberg EP, Iglewski BH (1999) Quinolone signaling in the cell-to-cell communication system of Pseudomonas aeruginosa. Proc Natl Acad Sci U S A 96(20):11229-11234

Radecka I, Irorere V, Jiang G, Hill D, Williams C, Adamus G, Kwiecień M, Marek AA, Zawadiak J, Johnston B, Kowalczuk M (2016) Oxidized polyethylene wax as a potential carbon source for PHA production. Materials 9(5):367. https://doi.org/10.3390/ma9050367

Rutherford ST, Bassler BL (2012) Bacterial quorum sensing: its role in virulence and possibilities for its control. Cold Spring Harb Perspect Med 2(11):a012427. https://doi.org/10.1101/cshperspect.a012427

Smyth TJ, Rudden M, Tsaousi K, Marchant R, Banat IM (2014) Protocols for the detection and chemical characterisation of microbial glycolipids. In: McGenity T, Timmis K, Nogales B (eds) Hydrocarbon and lipid microbiology protocols. Springer Protocols Handbooks. Springer, Berlin, Heidelberg, pp 29-60

Studer SV, Mandel MJ, Ruby EG (2008) AinS quorum sensing regulates the Vibrio fischeri acetate switch. J Bacteriol 190(17):5915-5923

Tiso T, Zauter R, Tulke H, Leuchtle B, Li W-J, Behrens B, Wittgens A, Rosenau F, Hayen H, Blank LM (2017) Designer rhamnolipids by reduction of congener diversity: production and characterization. Microb Cell Factories 16(1):225. https://doi.org/10.1186/s12934017-0838-y

Torabi Delshad S, Soltanian S, Sharifiyazdi H, Bossier P (2018) Effect of quorum quenching bacteria on growth, virulence factors and biofilm formation of Yersinia ruckeri in vitro and an in vivo evaluation of their probiotic effect in rainbow trout. J Fish Dis 41(9):1429-1438. https://doi.org/10.1111/jfd.12840

Tripathi L, Wu L-P, Chen J, Chen G-Q (2012) Synthesis of diblock copolymer poly-3-hydroxybutyrate-block-poly-3hydroxyhexanoate [PHB- $b$-PHHx] by a $\beta$-oxidation weakened Pseudomonas putida KT2442. Microb Cell Factories 11(1):44. https://doi.org/10.1186/1475-2859-11-44

Tseng BS, Majerczyk CD, Passos da Silva D, Chandler JR, Greenberg EP, Parsek MR (2016) Quorum sensing influences Burkholderia thailandensis biofilm development and matrix production. J Bacteriol 198(19):2643-2650. https://doi.org/10.1128/jb.00047-16

Ulrich RL, Deshazer D, Brueggemann EE, Hines HB, Oyston PC, Jeddeloh JA (2004) Role of quorum sensing in the pathogenicity of Burkholderia pseudomallei. J Med Microbiol 53(11):1053-1064

Williams P (2007) Quorum sensing, communication and cross-kingdom signalling in the bacterial world. Microbiology 153(12):3923-3938. https://doi.org/10.1099/mic.0.2007/012856-0

Xu C, Li M, Huang Y, Zhang Z, Bian Z, Song S (2011) Involvement of quorum-sensing in biosynthesis of polyhydroxyalkanoates in Pseudomonas aeruginosa. Wei Sheng Wu Xue Bao 51(6):769-775

Zulianello L, Canard C, Kohler T, Caille D, Lacroix JS, Meda P (2006) Rhamnolipids are virulence factors that promote early infiltration of primary human airway epithelia by Pseudomonas aeruginosa. Infect Immun 74(6):3134-3147

Publisher's note Springer Nature remains neutral with regard to jurisdictional claims in published maps and institutional affiliations. 